\title{
RE: Hypothalamic Pituitary Adrenocortical Axis Suppression following a Single Epidural Injection of Methylprednisolone Acetate: Review, Patient Safety and Clinical Care
}

\section{TO THE EDITOR:}

The recent prospective study, "Hypothalamic Pituitary Adrenocortical Axis Suppression following a Single Epidural Injection of Methylprednisolone Acetate" by Abdul A, Ghai B, Bansai D et al (1), provides valuable insight into the immediate effects of a single dose of methylprednisolone on systemic cortisol and adrenocorticotropic hormone levels in the human body. The study was thorough, informative, and adequately powered. This knowledge reinforces our current understanding of the effects of commonly used steroids in the practice of pain management and provides an opportunity for improved patient care and safety.

In the 30 patients enrolled in the study, there was a considerable decrease in serum cortisol levels on days $7(72.99 \mathrm{nmol} / \mathrm{L})$ and $14(194.45 \mathrm{nmol} / \mathrm{L})$ post-injection compared to a pre-injection median baseline of 329.55 $\mathrm{nmol} / \mathrm{L}$. For post procedure patient safety and improved clinical care, we recommend written discharge instructions given to patients to include the type of steroid, quantitative amount, date and site of injection. The rationale being, should the patient require an emergent surgery during the 2 to 4 weeks post injection of steroid, the patient might not be able to handle this stress without additional steroid coverage. This study also showed serum cortisol levels did not return to baseline until 4 weeks after the single steroid injection. Therefore, it is advisable to receive additional steroid coverage during the perioperative period should surgery be necessary within up to 4 weeks following a single dose of an epidural steroid injection.

We encourage an additional study assessing the degree of adrenal suppression with dexamethasone as the epidural steroid injectate. In the study by Schnei$\operatorname{der}$ A. et al (2), concerning adverse events have been reported when particulate corticosteroids e.g. methylprednisolone, triamcinolone, were used as injectate for epidural injections. Embolic effects associated with particulate corticosteroids have resulted in spinal cord infarction, cerebellar and brainstem infarction, cortical blindness, and even death. Current commercially available corticosteroids include methylprednisolone ac- etate, triamcinolone acetate, betamethasone acetate / phosphate, and dexamethasone phosphate. The first 3 corticosteroids exist either as large particulates or aggregates, being defined as particles that exceed the size of a typical red blood cell $(6-8 \mu \mathrm{m})$. It is theorized that these large particulates correlate with adverse events. Of those listed, dexamethasone phosphate is the only non-particulate, non-aggregate forming corticosteroid. Given the issues with particulate steroids, the majority of pain practices have shifted toward usage of non-particulate steroids for epidural injections. We recommend that first-line steroid choice for epidural injections include soluble, non-particulate steroids such as dexamethasone. We suggest the authors repeat their well-designed study using dexamethasone instead of methylprednisolone to identify the degree of Hypothalamic Pituitary Adrenocortical (HPA) Axis suppression following a single dose injection and additionally, to compare the degree of HPA Axis suppression between epidural methylprednisolone and dexamethasone.

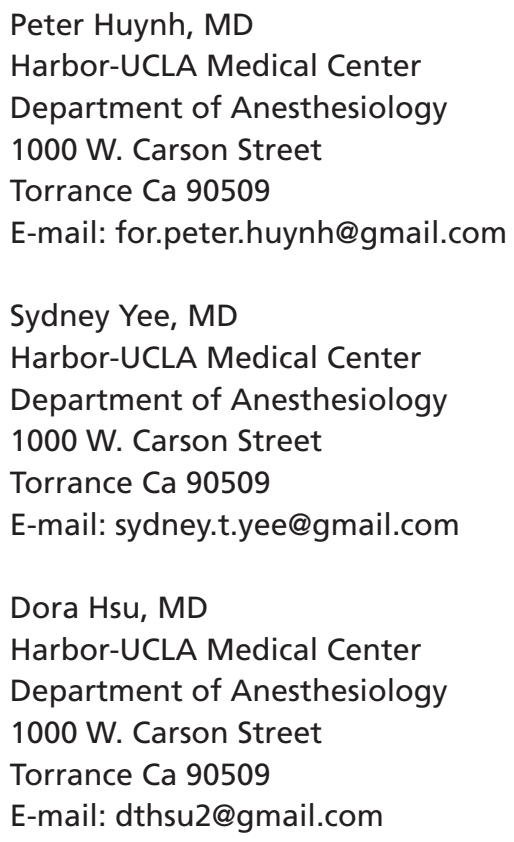




\section{References}

1. Abdul A, Ghai B, Bansal D, Sachdeva N, Bhansali A, Dhatt SS. Hypothalamic pituitary adrenocortical axis suppression following a single epidural injection of methylprednisolone acetate. Pain Physician 2017; 20:E991-E1001.

2. Schneider A, Varghis N, Kennedy D. Ideal corticosteroid choice for epidural steroid injections: A review of safety and efficacy. Curr Phys Med Rehabil Reports 2015; 3:151158. 